\title{
Thermal effects in a triplet and beam interaction in a plasma
}

Cite as: Phys. Plasmas 26, 113101 (2019); https://doi.org/10.1063/1.5125272

Submitted: 21 August 2019. Accepted: 14 October 2019. Published Online: 01 November 2019

E. Peter, R. Pakter, F. B. Rizzato, and S. Marini

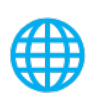

View Online

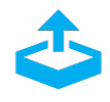

Export Citation

\section{ARTICLES YOU MAY BE INTERESTED IN}

Hybrid particle-in-cell simulations of laser-driven plasma interpenetration, heating, and entrainment

Physics of Plasmas 26, 112107 (2019); https://doi.org/10.1063/1.5110512

Engineered hyperuniformity for directional light extraction

APL Photonics 4, 110801 (2019); https://doi.org/10.1063/1.5124302

Chromatic matching in a plasma undulator

Physics of Plasmas 26, 113102 (2019); https://doi.org/10.1063/1.5120868

\section{AIP Advances Fluids and Plasmas Collection}




\title{
Thermal effects in a triplet and beam interaction in a plasma
}

\author{
Cite as: Phys. Plasmas 26, 113101 (2019); doi: 10.1063/1.5125272 \\ Submitted: 21 August 2019 . Accepted: 14 October 2019 . \\ Published Online: 1 November 2019
}

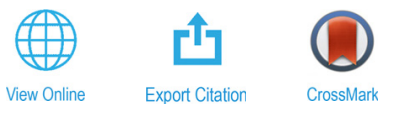

\author{
E. Peter, ${ }^{1, a)}$ R. Pakter, ${ }^{1, a)}$ F. B. Rizzato, ${ }^{1, a)}$ and S. Marini ${ }^{2, b)}$
}

\author{
AFFILIATIONS \\ 'Instituto de Física, Universidade Federal do Rio Grande do Sul, Caixa Postal 15051, 91501-970 Porto Alegre, RS, Brasil \\ ${ }^{2}$ LULI, Sorbonne Université, CNRS, École Polytechnique, CEA, Université Paris-Saclay, F-75252 Paris cedex 05, France \\ a)Electronic addresses: peterpeter@uol.com.br; pakter@if.ufrgs.br; and rizzato@if.ufrgs.br \\ b) marini@ufrgs.br
}

\begin{abstract}
The dynamics of three interacting waves fulfilling the frequency and wavenumber matching conditions can be dramatically modified if a charged particle beam is added to the system. Energy is exchanged not only among the waves but also between the particles of the beam and the waves. In the present work, a model that includes these interactions is revisited, allowing a more realistic case where the beam has an initial velocity distribution. This study aims to present the role of this thermal effect over the system at the beginning of the dynamics and after the breakdown of the laminar regime of the particle beam.
\end{abstract}

Published under license by AIP Publishing. https://doi.org/10.1063/1.5125272

\section{INTRODUCTION}

The study of a conservative system formed by three waves interacting with themselves was the subject of many works. ${ }^{1-12}$ The interaction takes place, according to the wave triplet concept, when resonant conditions are met: there must be frequency and wavelength matching conditions (the sum or difference of the frequency/wavelength of two waves is equal to the frequency/wavelength of the third wave).

A lot of interesting results come from this theoretical proposal. For example, if the interaction parameter (which depends on the plasma frequency) is small enough, the frequency of the envelope of each wave is slower than the slowest frequency of the carriers. In this case, the dynamics of the envelope of each wave is well described by a periodic and regular function and a modulational approximation may be applied to describe the evolution of the system. Moreover, due to frequency and wavenumber matching conditions and due to energy conservation, constants of motion are found. , $^{212-14}$

However, waves can also interact with charged particles, exchanging energy, in a plasma, for example. The interaction between a nonrelativistic charged particle beam and both electrostatic and electromagnetic waves in a plasma was studied in many works. In the present work, we are particularly interested in Refs. 15 and 16. In Ref. 15, the particles of the beam were able to interact with the waves, but, as no frequency and wavenumber matching conditions were allowed, the interaction between the waves was neglected. In that work, the authors showed that in a system composed of transversal and longitudinal modes, the amplitudes of the longitudinal modes can grow exponentially. The exponential growth of a mode and the way the phase-space evolves along the time have, indeed, similarities with one-dimensional free-electron lasers (FELs). ${ }^{17-21}$ Some of those similarities were explored in Ref. 16.

The interaction between the triplet and the particle beam, even for very small values of the frequency of plasma, was shown to change dramatically the dynamics of the waves, and for this reason, it must be taken into account in order to obtain more realistic results. As seen in Ref. 16, the regular dynamics of the envelopes is completely destroyed by the interaction: the longitudinal mode is excited, growing exponentially. After saturation, the amplitude of the longitudinal mode begins to oscillate around a mean value with two characteristic frequencies: the highest one is related to the frequency of the plasma, while the lowest one is related to the frequency of the triplet (in the case of no particles). The amplification of the electrostatic mode may have applications in accelerator devices. ${ }^{22-24}$ For example, the acceleration can be obtained for a second beam (the density of this beam must be much smaller than the first beam in order to promote nothing more than a perturbation on the original system). Under proper conditions (where the proper conditions include the initial velocity of the particles, phase velocity of the electrostatic field, and amplitude and length modulation of the field), a particle of the second beam reaches the phase velocity associated with the electrostatic field. As seen in Ref. 22, at this moment, the particle is accelerated by the field. 
Thermal effects due to the initial energy distribution of the particles of the beam are not avoidable and must be considered as well. The presence of an initial velocity distribution introduces a new component in the system dynamics that can impose obstacles to the applications and devices coming from the theory. The understanding of the effect of the initial velocity distribution in this theoretical model is the target of the present work.

In this paper, we present the physical model in Sec. II, through a nonrelativistic dimensionless Lagrangian. In Sec. III, we describe how simulations were performed and we show the main results of this research, while in Sec. IV, we drawn our conclusions.

\section{PHYSICAL MODEL}

The physical model of this work is exactly the same as Ref. 16 and based on Ref. 15. Here, a nonrelativistic beam propagating along the $x$-direction interacts with longitudinal, copropagating, and counterpropagating transversal waves. The interaction among the particles of the beam as well as the triplet interaction between the waves is considered. Moreover, the particles of the beam are allowed to have an initial velocity distribution (and the role of the initial distribution is the focus of the present study).

Longitudinal, copropagating, and counterpropagating transversal waves interact, in a cold plasma, with a nonrelativistic particle beam with an initial velocity distribution.

The full Lagrangian that describes the model is expressed as

$$
\begin{aligned}
L= & \int d^{3} x\left[\frac{1}{2} m n|\vec{v}|^{2}+\frac{1}{2} m \frac{\rho}{e}|\vec{v}|^{2}\right] \\
& +\frac{1}{8 \pi}\left[d^{3} x\left(\left|\nabla \Phi+\frac{1}{c} \frac{\partial \vec{A}}{\partial t}\right|^{2}-|\vec{\nabla} \times \vec{A}|^{2}\right)+\int d^{3} x\left(\frac{1}{c} \vec{j} \cdot \vec{A}-\rho \Phi\right)\right. \\
& +\sum_{i=1}^{N}\left[\frac{1}{2} m\left|\dot{\vec{r}}_{i}\right|^{2}+e \Phi\left(\vec{r}_{i}, t\right)-\frac{e}{c} \dot{\vec{r}}_{i} \cdot \vec{A}\left(\vec{r}_{i}, t\right)\right]
\end{aligned}
$$

where $n$ is the particle density of the background plasma (which we consider as a constant), $\rho(\vec{r}, t)$ is the local fluctuation of charge density of the background plasma, $\vec{v}(\vec{r}, t)$ is the Eulerian velocity, $\Phi(\vec{r}, t)$ is the scalar potential, $\vec{A}(\vec{r}, t)$ is the total vector potential, $\vec{j}(\vec{r}, t)$ is the current, $m$ is the mass of the electron, $\vec{r}_{i}(t)$ and $\dot{\vec{r}}_{i}(t)$ are the position and the velocity of the $i$-particle of the beam, and $e$ is the charge of the electron.

The fields of the longitudinal $(\Phi)$ and the transversal $\left(\vec{A}_{1}\right.$ is a copropagating and $\vec{A}_{2}$ is a counterpropagating) waves are described by the following potentials, respectively,

$$
\begin{aligned}
\Phi & =\phi(t) \cos \left[k_{L} x-\omega_{L} t-\beta(t)\right], \\
\vec{A}_{1} & =a_{1}(t) \cos \left[-k_{1} x+\omega_{1} t-\theta_{1}(t)\right] \hat{\mathbf{e}}, \\
\vec{A}_{2} & =a_{2}(t) \cos \left[-k_{2} x-\omega_{2} t-\theta_{2}(t)\right] \hat{\mathbf{e}}
\end{aligned}
$$

with the fields written in terms of the product of slowly varying amplitudes $\left[\phi(t), a_{1}(t)\right.$ and $\left.a_{2}(t)\right]$ and the cosine of the fast phases. Inside of the cosines, there are slow quantities $\left[\beta(t), \theta_{1}(t)\right.$ and $\left.\theta_{2}(t)\right]$ related to the evolution of the envelope of the waves. We assumed the field with respect to Whitham's envelope approximation. We identify the longitudinal wave to be a plasma wave, where $\omega_{L}=\omega_{p}$, while the total vector potential is $\vec{A}=\vec{A}_{1}+\vec{A}_{2}$ and the polarization versor is given by $\hat{\mathbf{e}}$. The fixed sinelike shape of the potentials imposes that the density of charge in the system must be limited. Otherwise, the selfconsistent fields produced by the charged particles could modify this shape.

The matching condition of the frequency is $\omega_{1}=\omega_{2}+\omega_{p}$, with $\omega_{p}^{2}=4 \pi n e^{2} / m$ being the plasma frequency. Moreover, using that for a cold plasma, the dispersion relation for the electromagnetic waves is described by $\omega_{i}^{2}=\omega_{p}^{2}+k_{i}^{2} c^{2}$; we find that the wavelength relation between the waves is written as $k_{L}=k_{1}+k_{2}$.

Considering $\omega_{1}=\alpha \omega_{p}$, where $\alpha>1, \omega_{2}=(\alpha-1) \omega_{p}, k_{1}=\omega_{p}$ $\sqrt{\alpha^{2}-1} / c$, and $k_{2}=\omega_{p} \sqrt{(\alpha-1)^{2}-1} / c$, the resonance velocity of the beam is given by $v_{r}=\omega_{p} / k_{L}=c /\left(\sqrt{\alpha^{2}-1}+\sqrt{(\alpha-1)^{2}-1}\right)$. As can be seen, if we increase $\alpha, v_{r}$ becomes smaller.

The linear relations for the background plasma are maintained the same as Refs. 15 and 16,

$$
\begin{aligned}
\vec{v}_{\|} & =\frac{1}{4 \pi n e} \frac{\partial \nabla \Phi}{\partial t}, \\
\vec{v}_{\perp} & =\frac{e}{m c} \vec{A}, \\
\vec{j}_{\|} & =-e n \vec{v}_{\|}, \\
\vec{j}_{\perp} & =-e n \vec{v}_{\perp}, \\
\rho & =-\frac{1}{4 \pi} \nabla^{2} \Phi,
\end{aligned}
$$

where $\vec{v}_{\|}$and $\vec{v}_{\perp}$ are the longitudinal and transverse velocities.

Assuming periodic boundary conditions, Eqs. (2) and (3) are substituted into Eq. (1). Then, averaging in space the Lagrangian (using a box of size $l$ ), we obtain

$$
\begin{aligned}
L= & \sum_{i=1}^{N} \frac{1}{2} m\left(\dot{x}_{i}^{2}+\dot{y}_{i}^{2}\right)+e \sum_{i=1}^{N} \phi \cos \left(k_{L} x_{i}-\omega_{p} t-\beta\right) \\
& -\frac{e}{c} \sum_{i=1}^{N} \dot{y}_{i}\left[a_{1} \cos \left(-k_{1} x_{i}+\omega_{1} t-\theta_{1}\right)\right] \\
& -\frac{e}{c} \sum_{i=1}^{N} \dot{y}_{i}\left[a_{2} \cos \left(-k_{2} x_{i}-\omega_{2} t-\theta_{2}\right)\right] \\
& +\frac{l^{3} \dot{\beta}}{8 \pi \omega_{p}} \phi^{2}+\frac{l^{3}}{8 \pi c^{2}}\left(-\omega_{1} \dot{\theta}_{1} a_{1}^{2}+\omega_{2} \dot{\theta}_{2} a_{2}^{2}\right) \\
& +\frac{l^{3} e}{32 \pi m c^{2}} k_{L}^{2} \phi a_{1} a_{2} \cos \left(-\beta-\theta_{1}-\theta_{2}\right) .
\end{aligned}
$$

Equation (4) is rewritten in terms of dimensionless variables,

$$
\begin{aligned}
L= & \frac{1}{2}\left(\frac{2 n_{b}}{n}\right)\left[\left\langle\dot{\xi}_{i}^{2}\right\rangle+\left\langle\dot{\eta}_{i}^{2}\right\rangle\right]+\left(\frac{2 n_{b}}{n}\right) \sqrt{J}\left\langle\cos \left(\xi_{i}-\beta\right)\right\rangle \\
& -\left(\frac{2 n_{b}}{n}\right) \sqrt{\frac{\omega_{p} I_{1}}{\omega_{1} \mu_{1}}}\left\langle\dot{\eta}_{i} \cos \left(\mu_{1}\left[\xi_{i}+\theta_{1}\right]\right)\right\rangle \\
& +\left(\frac{2 n_{b}}{n}\right) \sqrt{\frac{\omega_{p} I_{2}}{\omega_{2} \mu_{2}}}\left\langle\dot{\eta}_{i} \cos \left(\mu_{2}\left[\xi_{i}+\theta_{2}\right]\right)\right\rangle \\
& +\dot{\beta} J+\left(1-\frac{\omega_{1}}{\mu_{1} \omega_{p}}-\dot{\theta}_{1}\right) I_{1}+\left(-1-\frac{\omega_{2}}{\mu_{2} \omega_{p}}+\dot{\theta}_{2}\right) I_{2} \\
& +\frac{1}{4} \sqrt{\frac{\omega_{p}^{2} I_{1} I_{2} J}{\omega_{1} \omega_{2} \mu_{1} \mu_{2}}} \cos \left(\beta+\mu_{1} \theta_{1}+\mu_{2} \theta_{2}\right),
\end{aligned}
$$


where

$$
\begin{aligned}
\xi_{i} & =k_{L} x_{i}-\omega_{p} t, \\
\eta_{i} & =k_{L} y_{i}, \\
t & =\omega_{p} t, \\
\phi^{2} & =\left(\frac{m \omega_{p}^{2}}{e k_{L}^{2}}\right)^{2} J, \\
a_{j}^{2} & =\left(\frac{m c \omega_{p}}{e k_{L}}\right)^{2}\left(\frac{\omega_{p}}{\omega_{j}}\right) \frac{I_{j}}{\mu_{j}}, \\
\mu_{1} \theta_{1} & =\theta_{1}+\mu_{1} \omega_{p} t-\omega_{1} t, \\
\mu_{2} \theta_{2} & =\theta_{2}+\mu_{2} \omega_{p} t+\omega_{2} t, \\
\mu_{j} & =\frac{k_{j}}{k_{L}} \\
n_{b} & =\frac{N}{l^{3}} .
\end{aligned}
$$

$N$ is the number of particles. The brackets indicate a mean value over the particle beam distribution, i.e., $\langle x\rangle=\sum_{i=1}^{N} x_{i} / N$.

Equations of the evolution of the system are obtained from the Lagrangian written in Eq. (5). This Lagrangian is identical to the dimensionless Lagrangian of Ref. 16. In the present work, we allow the particle beam to have an initial velocity distribution (initial conditions of the particles).

\section{THERMAL EFFECTS}

An initial velocity distribution affects the dynamics of systems where charged beams interact with electromagnetic fields. This is the case, for example, for free electron lasers and beam transport. ${ }^{19,21,25-27}$ In FELs, the growth rate of the laser is reduced as the initial velocity spread increases. Moreover, the particles of the beam can be exactly represented by adiabatic fluid equations, while the dynamics of the particles is laminar in the phase-space velocity $\times$ position. If the velocity difference between the beam and the phase velocity of the ponderomotive wave is larger than the velocity spread, then the regime is called hydrodynamic and the initial velocity spread acts like a pressure term over the particles. ${ }^{28}$ However, as the velocity spread becomes larger than the difference between the beam and the phase velocity of the ponderomotive wave, the efficiency (gain) of the system decreases significantly. ${ }^{19-21,29,30}$

In the case of Ref. 16, in the absence of the triplet interaction, the growth rate of the longitudinal mode should be reduced as the initial velocity spread increases, as well as the amplitude of the longitudinal mode at the moment of the breakdown of the laminar regime (in the phase-space). This result is expected according to Refs. 19 and 21.

In this work, simulations are run to evaluate the role of the initial velocity distribution over the present given model. For this, Euler-Lagrange equations from the Lagrangian of Eq. (5) are numerically solved, considering a water-bag distribution for the initial velocity distribution of the particles of the beam (where $v_{\xi, 0}$ is the center and $\Delta v_{\xi, 0}$ is the half width of the distribution; thus, the initial velocity distribution is homogeneously distributed between $v_{\xi, 0}-\Delta v_{\xi, 0}$ and $\left.v_{\xi, 0}+\Delta v_{\xi, 0}\right)$.

If the initial distribution is not perfectly homogeneous in space and in velocity, the dynamics of the system is changed and the time scales are affected. Even for a random initial distribution, the finite number of particles generates local fluctuations of charge, for example. To circumvent this problem, the initial distribution is divided into layers. The total velocity width of the initial distribution is split equidistantly, where each layer has a single value of velocity and a homogeneous spatial distribution. This kind of strategy is commonly entitled as quite start, and it is a technique used to reduce noise without increasing the number $N$ of particles. ${ }^{31,32}$ For this work, $N=10000$ particles were taken in order to perform the simulations (100 velocity layers with 100 particles each).

The analysis of the role of the initial velocity distribution is made in two different situations. First, in Sec. III A, the focus is on the beginning of the dynamics: the system is studied until the breakdown of the laminar regime (which will be defined later); and then, in Sec. III B, the focus is on the dynamics after the breakdown of the laminar regime.

\section{A. Breakdown of the laminar regime}

If the triplet interaction is turned off, as one of the cases studied in Ref. 16, the growth rate (that is exponential) of the longitudinal mode is a function of the initial velocity of the beam. This result is similar to the result obtained from the one-dimensional high gain FEL theory. ${ }^{18}$ Introducing an initial velocity distribution to the beam, it is seen in Ref. 19 that the growth rate is a function of the center and, of course, of the width of the distribution.

In the hydrodynamical regime, the time until the breakdown of the laminar regime (the regime is considered laminar as long as each layer persists a single value function in the phase-space $v_{\xi} \times \xi$; for this reason, the phase-space is explored despite the Lagrangian formulation) does not depend strongly on the initial distribution width. However, as the width of the initial distribution increases, the growth rate decreases.

Moreover, in the case where the triplet interaction is turned off, the amplitude of $J$ at the moment of the breakdown of the laminar regime $\left(J_{b}\right)$ decreases as the initial distribution width increases. On the other hand, the value of the center of the initial distribution to maximize the value of $J_{b}$ for a fixed distribution width increases as the distribution width increases. Additionally, when the velocities of the particles are away from resonance, there is no breakdown of the laminar regime (and consequently no growth of $J$ ).

When the triplet interaction is taken into account, the growth rate is not linear (where linear means the amplitude grows exponentially with time) anymore, and despite the action of the triplet interaction, in the beginning of the dynamics of the system, the particles of the nonrelativistic charged beam evolve like in a laminar regime.

At the moment of the breakdown of the laminar regime, the amplitude of $J$ is evaluated and the result is given in Fig. 1. The figures of this paper were built using the following set of parameters: $\omega_{1}$ $=20, \omega_{p}=1(\alpha=20)$ and the following initial conditions: $\dot{\xi}(t=0)$ $=0, I_{1}(t=0)=2, I_{2}(t=0)=2$ and $J(t=0)=10^{-4}, \quad \beta(t=0)$ $=\theta_{1}(t=0)=\theta_{2}(t=0)=0$, unless otherwise stated. The colored solid lines are plotted for different values of $\Delta v_{\xi, 0}$ [in panel (a), $n_{b} / n=0.05$, while in panel (b), $n_{b} / n=0.20$ ]. As can be seen, the amplitude decreases as the center of the distribution decreases. There is an important point, located at $v_{\xi, 0} \approx-0.1$ in panel (a) and $v_{\xi, 0}$ $\approx-0.30$ in panel (b) in which $J_{b}$ seems to be independent of the distribution width. 


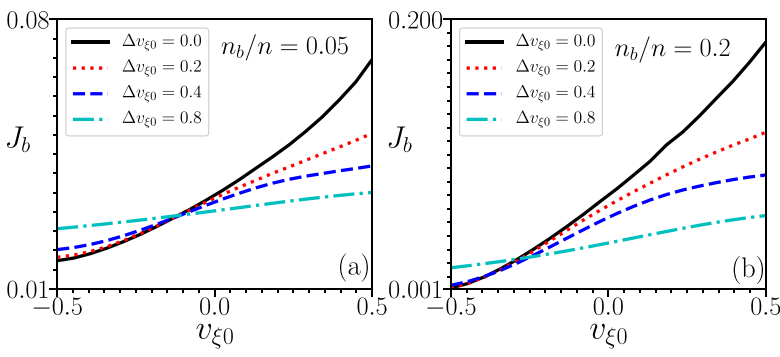

FIG. 1. Amplitude $J$ evaluated at the moment of the breakdown of the laminar regime as a function of the center of the distribution for different initial distribution widths, using the following parameters: $\omega_{1}=20, \omega_{p}=1(\alpha=20)$ and the following initial conditions $\dot{\xi}(t=0)=0, I_{1}(t=0)=2, I_{2}(t=0)=2$ and $J(t=0)$ $=10^{-4}, \beta(t=0)=\theta_{1}(t=0)=\theta_{2}(t=0)=0$. In panel $(\mathrm{a}), n_{b} / n=0.05$, while in panel (b), $n_{b} / n=0.20$.

The increase in $J_{b}$, while $v_{\xi, 0}$ is increased, may appear quite surprising. But Fig. 1 cannot be analyzed alone. In Fig. 2 is shown the time until the breakdown of the laminar regime as a function of the center of the distribution for $n_{b} / n=0.05$ in panel (a) and $n_{b} / n$ $=0.20$ in panel (b). The time until the breakdown seems to be independent of the value of the initial spread for $v_{\xi, 0} \approx-0.10$ in panel (a) and $v_{\xi, 0} \approx-0.30$ in panel (b). We call these points $v_{\xi, \text { in }}$. Near $v_{\xi, \text { in }}, t_{b}$ is weakly dependent on the center of the distribution and on the distribution width. As much as $v_{\xi, 0}$ becomes different from $v_{\xi, \text { in }}, t_{b}$ increases. When Figs. 1 and 2 are compared, it is clear that despite the increase in $t_{b}$ as $v_{\xi, 0}$ decreases, the growth rate of $J$ becomes smaller in a way that $J_{b}$ decreases as $v_{\xi, 0}$ decreases. The opposite occurs for $v_{\xi, 0}>v_{\xi, \text { in }}$ : as $v_{\xi, 0}$ increases, $t_{b}$ also increases. In this case, $t_{b}$ increases more than the decrease in the mean value of the growth rate of $J$. It explains why $J_{b}$ increases for $v_{\xi, 0}>v_{\xi, i n}$.

Detailed maps showing $J_{b}$ as a function of $v_{\xi, 0}$ and $\Delta v_{\xi, 0}$ are shown in Fig. 3 for $n_{b} / n=0.05$ in panel (a) and $n_{b} / n=0.20$ in panel (b). The colors represent the value of $J_{b}$, from light green $J_{b} \approx 0.01$ to dark red $J_{b} \approx 0.07$ in panel (a) and from light green $J_{b} \approx 0.0001$ to dark red $J_{b} \approx 0.20$ in panel (b). From the maps, it is found that $J_{b}$ increases as $\Delta v_{\xi, 0}$ goes to zero for a fixed $v_{\xi, 0}$ and increases as $v_{\xi, 0}$ increases for a fixed $\Delta v_{\xi, 0}$. The initial distribution acts
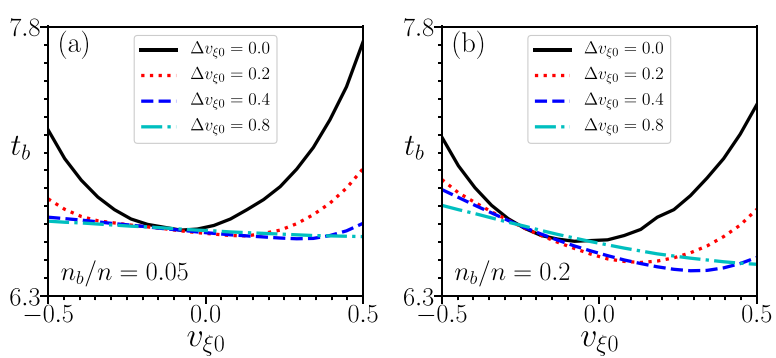

FIG. 2. Time until the breakdown of the laminar regime $t_{b}$ as a function of the center of the distribution for different initial distribution widths, using the following parameters: $\omega_{1}=20, \omega_{1}=20, \omega_{p}=1(\alpha=20)$ and the following initial conditions $\dot{\xi}(t=0)=0, I_{1}(t=0)=2, I_{2}(t=0)=2$ and $J(t=0)=10^{-4}, \beta(t=0)$ $=\theta_{1}(t=0)=\theta_{2}(t=0)=0$. In panel $(a), n_{b} / n=0.05$, while in panel $(b)$, $n_{b} / n=0.20$
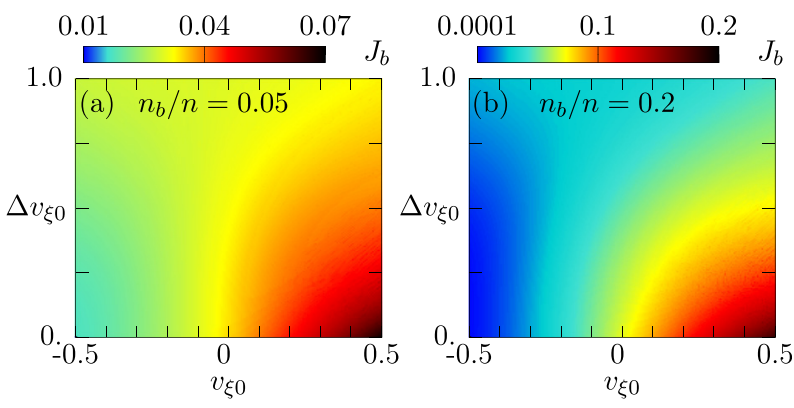

FIG. 3. Map of $J_{b}$ as a function of $\Delta v_{\xi, 0}$ in the vertical axis and $v_{\xi, 0}$ in the horizontal axis, using the following parameters: $\omega_{1}=20, \omega_{p}=1(\alpha=20)$ and the following initial conditions $\dot{\xi}(t=0)=0, l_{1}(t=0)=2, I_{2}(t=0)=2$ and $J(t=0)=10^{-4}, \beta(t=0)=\theta_{1}(t=0)=\theta_{2}(t=0)=0$. In panel (a), $n_{b} / n=0.05$, while in panel (b), $n_{b} / n=0.20$.

like a pressure term, reducing the growth rate of $J$. Moreover, as $n_{b} / n$ increases, $J_{b}$ also increases. This last result is expected, once as we increase $n_{b} / n$, we are putting more particles in the system, which implies that more energies are to be transferred to $J$.

Maps were also obtained for $t_{b}$. The results are shown in Fig. 4 . For panel (a), $n_{b} / n=0.05$, while for panel (b), $n_{b} / n=0.20$. The colors vary from light green $t_{b}=6.6$ to dark red $t_{b}=7.8$ in panel (a) and from light green $t_{b}=6.4$ to dark red $t_{b}=7.4$ in panel (b). As can be seen, $t_{b}$ is less dependent of $n_{b} / n$ in relation to $J_{b}$ for the parameters used in this work. Furthermore, as seen in Fig. 2, $t_{b}$ is almost uniform in the vicinity of $v_{\xi, \text { in }}$. The white solid line represents the value of $v_{\xi, 0}$ to minimize $t_{b}$ for a fixed $\Delta v_{\xi, 0}$ value. As the initial distribution width increases, $v_{\xi, 0}$ to minimize $t_{b}$ increases.

The way the breakdown of the laminar regime takes place in the phase space $v_{\xi} \times \xi$ depends on the distribution center and on the distribution width. In Fig. 5 is shown the phase-space at the moment of the breakdown (and just after) for different values of $\Delta v_{\xi, 0}$ and for $v_{\xi, 0}=-0.1$. In panel (a) $\left(\Delta v_{\xi, 0}=0.2\right)$, the initial distribution width is narrow. In this case, all the particles are exchanging energy with the triplet and the configuration of the phase-space at the moment of the breakdown of the laminar regime is quite similar to what happens for
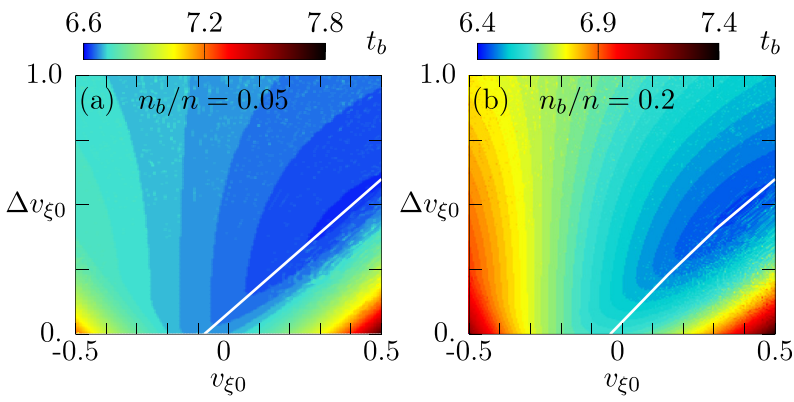

FIG. 4. Map of $t_{b}$ as a function of $\Delta v_{\xi, 0}$ in the vertical axis and $v_{\xi, 0}$ in the horizontal axis, using the following parameters: $\omega_{1}=20, \omega_{p}=1(\alpha=20)$ and the following initial conditions: $\dot{\xi}(t=0)=0, l_{1}(t=0)=2, I_{2}(t=0)=2$ and $J(t=0)$ $=10^{-4}, \beta(t=0)=\theta_{1}(t=0)=\theta_{2}(t=0)=0$. In panel $(\mathrm{a}), n_{b} / n=0.05$, while in panel (b), $n_{b} / n=0.20$. 

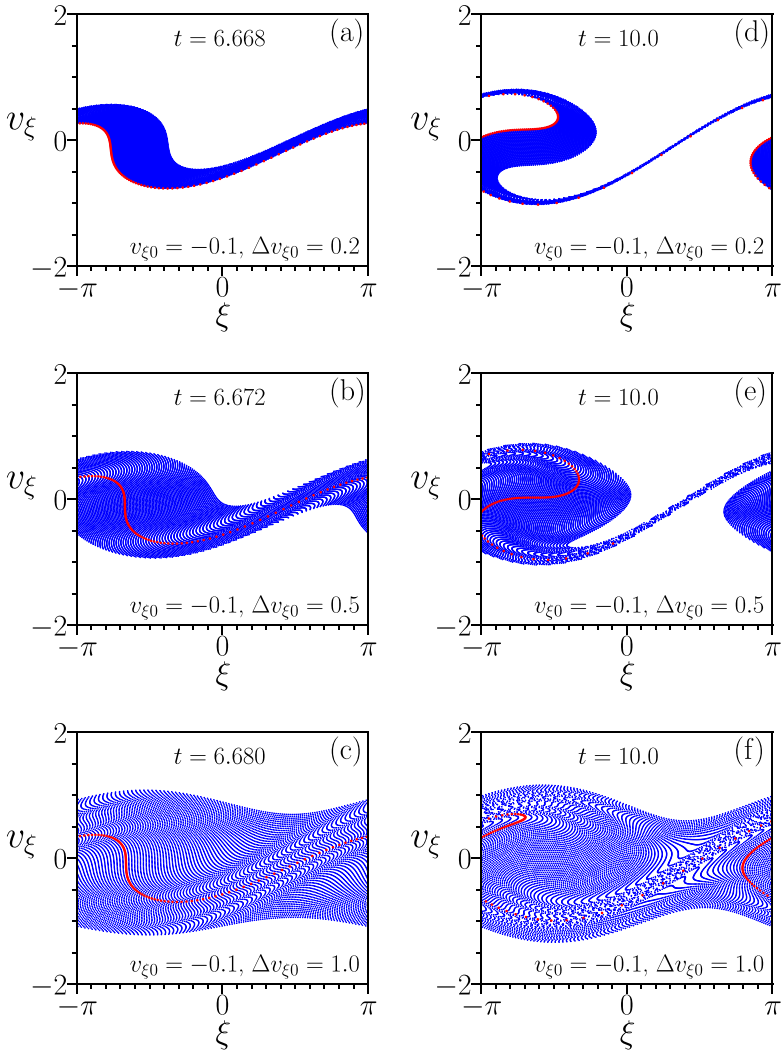

FIG. 5. Phase-space $v_{\xi} \times \xi$ for $v_{\xi, 0}=-0.1, n_{b} / n=0.05, \omega_{1}=20, \omega_{p}=1$ $(\alpha=20)$, and $\xi(t=0)=0$ and the following initial conditions: $l_{1}(t=0)=2$, $I_{2}(t=0)=2$ and $J(t=0)=10^{-4}, \beta(t=0)=\theta_{1}(t=0)=\theta_{2}(t=0)=0$. In panels (a) and (d) $\Delta v_{\xi, 0}=0.2$, panels (b) and (e) $\Delta v_{\xi, 0}=0.5$ and panels (c) and (f) $\Delta v_{\xi, 0}=1.0$.

a monoenergetic beam. Additionally, all the layers become a multi valued function in a small interval of time, as plotted in panel (d) for $t=10.00$.

In panel (b) of Fig. 5, the width of the distribution was increased. As can be seen, the first layer to become a multivalued function in the phase-space (represented by the red solid line) is not the bottom nor the upper layers. Besides it, the shapes of the bottom and the upper layers are clearly affected by the dynamics of the system, and eventually, they become multivalued functions as well, as shown in panel (e).

We built panel (c) increasing even more the width of the distribution. In this panel, the red solid line, which is the first layer to become a multivalued function, is internal to the distribution. Moreover, the upper and the bottom layers seem to be symmetrical in relation to the center of the initial distribution. This indicates that these layers are far from resonance and the particles in this region do not effectively exchange energy with the triplet. This result is represented in panel (f) for $t=10$.

An interesting result comes from varying the center of the initial distribution for the same value of the distribution width and analyzing the phase-space at $t_{b}$. Depending on the signal of the difference between the velocity of the center of the initial distribution and the resonant velocity, the way the breakdown of the laminar regime takes place is mirrored in relation to the resonant velocity.

Another way to understand the role of the initial distribution in the breakdown of the laminar regime is to evaluate the particle density of the beam along $-\pi<\xi<\pi$. In Fig. 6(a), comparison is made through three panels for different values of $\Delta v_{\xi, 0}$ (the same parameters used in Fig. 5). As the distribution width increases, the gap between the most and the fewest populated regions (in terms of $\xi$ ) decreases. This gap can be thought as an indirect measure of the fraction of the particles in resonance with the system. The referred result agrees with warm-beam FEL models in the literature ${ }^{19-21}$ and is somehow connected to the concept of bunch factor, applied in FELs. This suggests that the thermal effects act like a pressure over the particles.

Different from FELs, the bunching factor is a smooth function of the distribution width and of the beam density, while evaluated at the breakdown of the laminar regime (keeping fixed the center of velocity). In the FEL case, however, the laser grows due to the interaction among the particles and between the wave (laser) and the particles. Thus, there are cases in the FEL scenario where the laser does not grow, and for this reason, the bunching factor function does not vary smoothly as a function of density and distribution width. There is indeed a critical value of the distribution width beyond which there is no further breakdown of the laminar regime. On the contrary, in the present model, the interaction between the particles (triplet interaction) guarantees that the electrostatic field will grow and, consequently, will lead to the breakdown of the laminar regime. Moreover, in this case, the shape of the waves is kept sinusoidal: it does not allow huge gradients of particle's density.

\section{B. Role of the initial distribution in the evolution of $\mathbf{J}$}

In Sec. III A, the role of the initial velocity distribution until the breakdown of the laminar regime was on focus $\left(t_{b}, J_{b}\right.$, and the way the
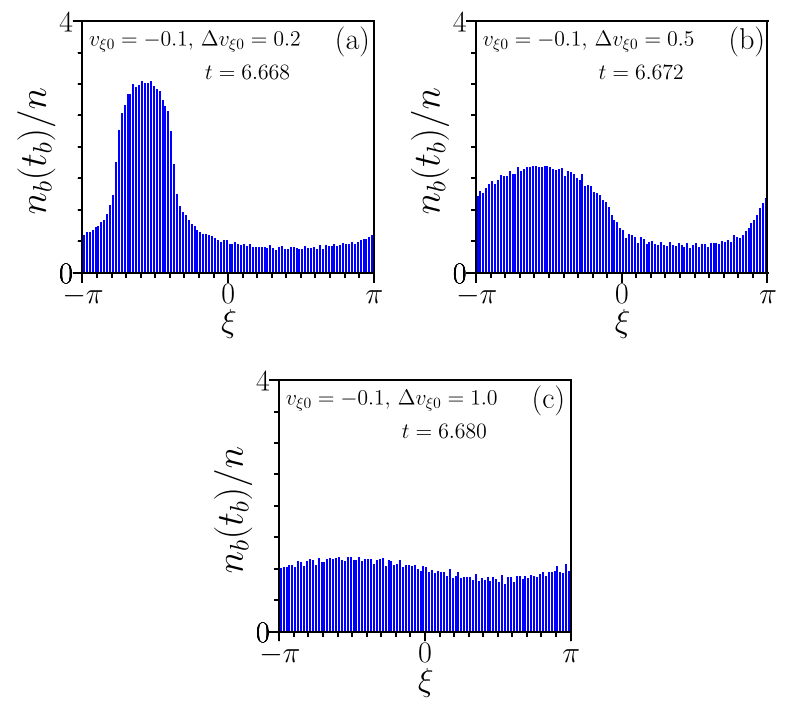

FIG. 6. Histogram of the spatial distribution of the particles at the moment of the breakdown of the laminar regime for $v_{\xi, 0}=-0.1, n_{b} / n=0.05, \omega_{1}=20, \omega_{p}$ $=1(\alpha=20), \dot{\xi}(t=0)=0$ and the following initial conditions: $l_{1}(t=0)$ $=2, I_{2}(t=0)=2$ and $J(t=0)=10^{-4}, \beta(t=0)=\theta_{1}(t=0)=\theta_{2}(t=0)$ $=0$. In panel (a) $\Delta v_{\xi, 0}=0.2$, panel (b) $\Delta v_{\xi, 0}=0.5$, and panel (c) $\Delta v_{\xi, 0}=1.0$. 
breakdown of the laminar regime takes place are functions of the initial distribution width and the center of the distribution). In Sec. III B, we discuss how the distribution affects the overall system evolution after the breakdown of the laminar regime.

For this, we plot in Fig. 7 the time evolution of $J$ in panel (a), $I_{1}$ in panel (b), and $I_{2}$ in panel (c), for $n_{b} / n=0.05, v_{\xi, 0}=-0.1$, and different values of $\Delta v_{\xi, 0}$. The value of the first peak of the fields has a strong dependence on the initial distribution width [different from $J_{b}$, as seen in Fig. 1(a), built using the same parameters]. We must point out that the low frequencies associated with the evolution of the fields are changed due to the distribution width as well. Moreover, as $\Delta v_{\xi, 0} \rightarrow \infty$, the evolution of the fields tends to be the case without the particle beam (red solid line).

Figure 8 was made increasing $n_{b} / n$ to $n_{b} / n=0.20$. In this case, the figure was built for $v_{\xi, 0}=-0.30$ and for different values of $\Delta v_{\xi, 0}$. As in Fig. 7, the peaks of the fields depend on the distribution width, different from $J_{b}$ in Fig. 1(b). The time interval was kept the same. As can be seen, the low frequencies depend on the initial distribution width and they were significantly increased in comparison to Fig. 7. This result was expected, once the increase in $n_{b} / n$ also increases the interaction term between the waves and consequently diminishes the period of oscillation of the triplet.

A way to estimate the frequency of the oscillations of the fields as a function of the initial distribution width is to plot a map with the time evolution of $J$ (through colors) against $\Delta v_{\xi, 0}$. It is exactly what was done in Fig. 9. Both in panel (a), made for $n_{b} / n=0.05$ and $v_{\xi, 0}=0$, and in panel (b), made for $n_{b} / n=0.20$ and $v_{\xi, 0}=0$, as the distribution width increases, the frequency of the oscillations of $J$ decreases. As seen in Figs. 7 and 8, if we increase the relation $n_{b} / n$, the frequency also increases.
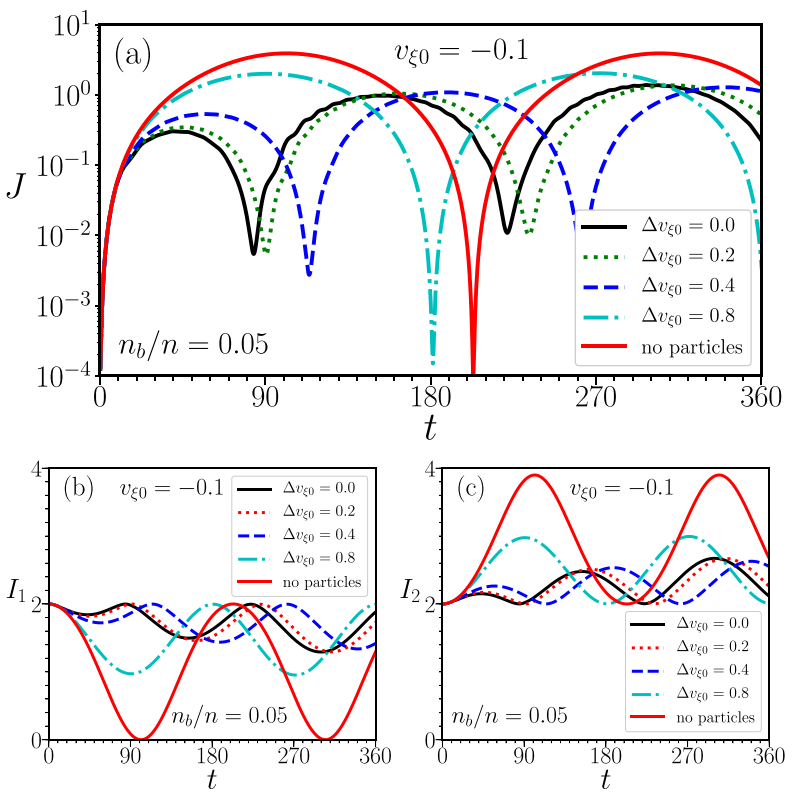

FIG. 7. Time evolution of the fields for $v_{\xi, 0}=-0.1, n_{b} / n=0.05, \omega_{1}=20$, $\omega_{p}=1(\alpha=20), \dot{\xi}(t=0)=0$ and the following initial conditions: $I_{1}(t=0)$ $=2, I_{2}(t=0)=2$ and $J(t=0)=10^{-4}, \beta(t=0)=\theta_{1}(t=0)=\theta_{2}(t=0)$ $=0 . J$ is plotted in panel (a), $I_{1}$ in panel (b), and $I_{2}$ in panel (c).
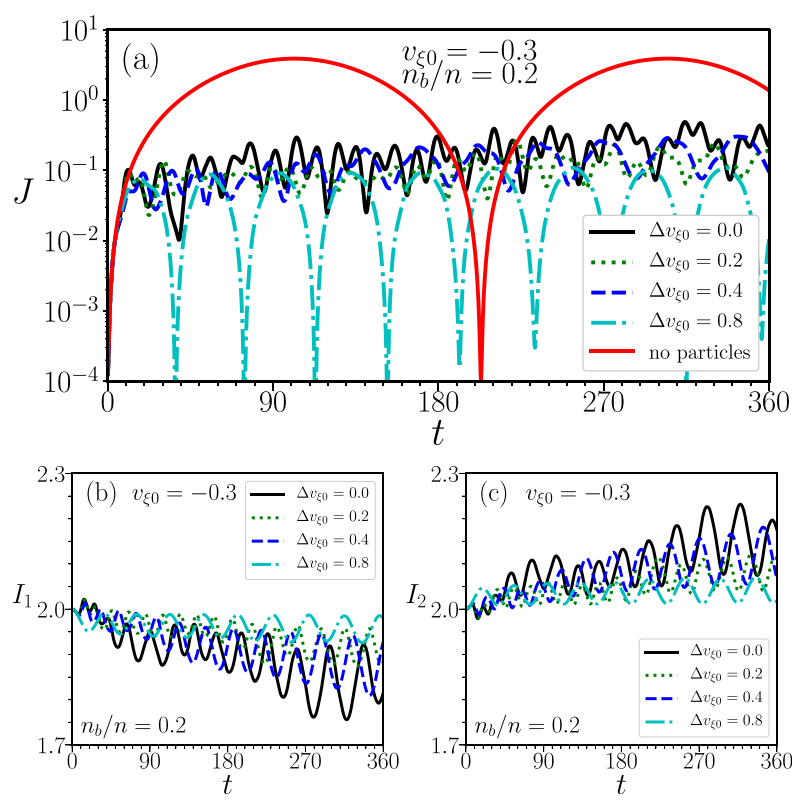

FIG. 8. Time evolution of the fields for $v_{\xi, 0}=-0.30, n_{b} / n=0.20, \omega_{1}=20$, $\omega_{p}=1(\alpha=20), \dot{\xi}(t=0)=0$ and the following initial conditions: $I_{1}(t=0)$ $=2, I_{2}(t=0)=2$ and $J(t=0)=10^{-4}, \beta(t=0)=\theta_{1}(t=0)=\theta_{2}(t=0)$ $=0 . J$ is plotted in panel $(\mathrm{a}), I_{1}$ in panel $(\mathrm{b})$, and $I_{2}$ in panel $(\mathrm{c})$.

\section{CONCLUSIONS}

In a previous work, ${ }^{16}$ it was shown that when an initially cold beam, even for a very small charge, is allowed to interact with a triplet, the dynamics of the system is completely changed. Besides the triplet interaction, the particles interact with the waves and with themselves. This kind of system emulates a more realistic model for a triplet composed of a plasma wave, where the particle beam can be thought as the free particles of the plasma.

However, free particles have different initial velocities. Thus, the inclusion of thermal effects (i.e., an initial velocity distribution) is needed. In the present work, a water-bag distribution for the initial velocities of the particles was used.

The dynamics of the particles in the phase-space (velocity against the position) is initially laminar. However, as the system evolves, some
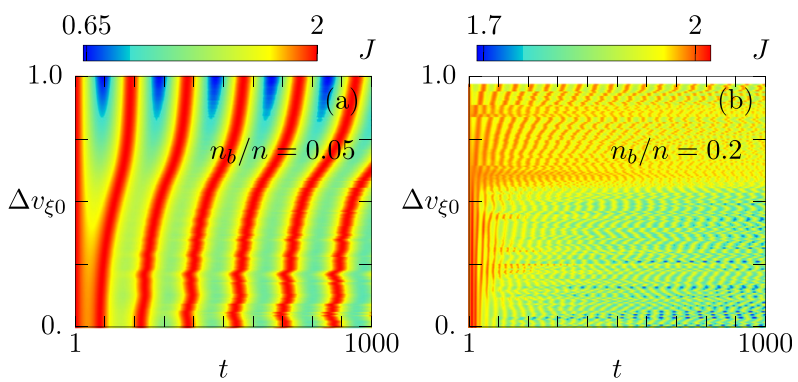

FIG. 9. Map of $J$ for $\omega_{1}=20, \omega_{p}=1(\alpha=20), \dot{\xi}(t=0)=0$ and the following initial conditions: $l_{1}(t=0)=2, l_{2}(t=0)=2$ and $J(t=0)=10^{-4}, \beta(t=0)$ $=\theta_{1}(t=0)=\theta_{2}(t=0)=0$. In panel $(\mathrm{a}), v_{\xi, 0}=0, n_{b} / n=0.05$, while in panel (b), $v_{\xi, 0}=0, n_{b} / n=0.20$. 
(or all) of the layers of the distribution become a multi valued function in the phase-space. We call the moment where the first layer becomes a multi valued function $t_{b}$ or the time of the breakdown of the laminar regime. The amplitude evaluated at this moment is called $J_{b}$. Both the center of the distribution and the distributions width affect $t_{b}$ and $J_{b}$. In general, if the distribution width increases, $J_{b}$ decreases (for a fixed $\left.v_{\xi, 0}\right)$ and if $v_{\xi, 0}$ increases, $J_{b}$ increases as well (for a fixed $\Delta v_{\xi, 0}$ ). The density of the particle beam also changes $J_{b}$ and $t_{b}$ since more particles can exchange energy with the waves.

When one looks at the phase space of the particles at $t_{b}$ or just after $t_{b}$, it is possible to understand some results. There are cases where all the layers of the distribution become a multi valued function in phase space and some of the layers become a multi valued function in phase space. Only particles near the resonant velocity will exchange, effectively, energy with the waves. In this sense, particles far from resonance act like a pressure over the others (reducing the growth rate of $J_{b}$ and increasing $t_{b}$ ). The initial distribution affects, also, the characteristic frequencies of the fields for $t \gg t_{b}$. As $\Delta v_{\xi, 0}$ increases, the frequency of the fields $J, I_{1}$, and $I_{2}$ also increases. Moreover, when $\Delta v_{\xi, 0} \rightarrow \infty$, the evolution of the fields becomes the same as the case of no particle beam in the system.

The present model is restricted to nonrelativistic velocities for the particles of the beam. Thus, a relativistic model should be developed. This will be the target of an upcoming paper based on Ref. 33 .

\section{ACKNOWLEDGMENTS}

This work was supported by CAPES and CNPq, Brazil, and by the Air Force Office of Scientific Research (AFOSR), USA, under Grant No. FA9550-16-1-0280. S.M. acknowledges support from Grant No. ANR-11-IDEX-0004-02 Plas@Par.

\section{REFERENCES}

${ }^{1}$ S. G. Thornhill and D. ter Haar, Phys. Rep. 43, 43 (1978).

${ }^{2}$ P. Iorra, S. Marini, E. Peter, R. Pakter, and F. B. Rizzato, Physica A 436, 686 (2015).

${ }^{3}$ P. K. Shukla, N. N. Rao, M. Y. Yu, and N. L. Tsintsadze, Phys. Rep. 138, 1 (1986).
${ }^{4} \mathrm{~J}$. Weiland and H. Wilhelmsson, Coherent Non-Linear Interaction of Waves in Plasmas (Pergamon, Oxford, 1977).

${ }^{5}$ T. Tajima and J. M. Dawson, Phys. Rev. Lett. 43, 267 (1979).

${ }^{6}$ G. Brodin and L. Stenflo, J. Plasma Phys. 79, 909 (2013).

${ }^{7}$ F. B. Rizzato and A. C.-L. Chian, J. Plasma Phys. 48, 71 (1992).

${ }^{8}$ P. M. Drysdale and P. A. Robinson, Phys. Plasmas 9, 4896 (2002).

${ }^{9}$ M. Frichembruder, R. Pakter, G. Gerhardt, and F. B. Rizzato, Phys. Rev. E 62, 7861 (2000).

${ }^{10}$ G. I. de Oliveira, L. P. L. de Oliveira, and F. B. Rizzato, Physica D 104, 119 (1997).

${ }^{11}$ G. I. de Oliveira, M. Frichembruder, and F. B. Rizzato, Physica D 164, 59 (2002).

${ }^{12}$ E. Peter, S. Marini, A. T. Chávez, and F. B. Rizzato, Physica A 463, 103 (2016).

${ }^{13}$ J. M. Manley and H. E. Rowe, Proc. IRE 44, 904 (1956).

${ }^{14}$ A. M. Batista, I. L. Caldas, S. R. Lopes, R. L. Viana, W. Horton, and P. J. Morrison, Phys. Plasmas 13, 042510 (2006).

${ }^{15}$ E. G. Evstatiev, W. Horton, and P. J. Morrison, Phys. Plasmas 10, 4090 (2003).

${ }^{16}$ E. Peter, S. Marini, R. Pakter, and F. B. Rizzato, Phys. Plasmas 24, 102124 (2017).

${ }^{17}$ R. Bonifacio, F. Casagrande, G. Cerchioni, L. de Salvo Souza, P. Pierini, and N. Piovella, Riv. Nuovo Cimento 13, 1 (1990).

${ }^{18}$ E. Peter, A. Endler, F. B. Rizzato, and A. Serbeto, Phys. Plasmas 20, 123104 (2013).

${ }^{19}$ E. Peter, A. Endler, and F. B. Rizzato, Phys. Plasmas 21, 113104 (2014).

${ }^{20}$ E. Peter, F. B. Rizzato, and A. Endler, J. Plasma Phys. 82, 905820307 (2016).

${ }^{21}$ E. Peter, F. B. Rizzato, and A. Endler, J. Plasma Phys. 83, 905830302 (2017).

${ }^{22}$ S. Marini, E. Peter, G. I. de Oliveira, and F. B. Rizzato, Phys. Plasmas 24, 093113 (2017)

${ }^{23}$ F. Russman, S. Marini, E. Peter, G. I. de Oliveira, and F. B. Rizzato, Phys. Plasmas 25, 023110 (2018).

${ }^{24}$ I. Almansa, D. Burton, R. A. Cairns, S. Marini, E. Peter, F. B. Rizzato, and F. Russman, Phys. Plasmas 25, 113107 (2018).

${ }^{25}$ S. Babaei and B. Maraghechi, Phys. Plasmas 15, 013102 (2008).

${ }^{26}$ A. Chakhmachi and B. Maraghechi, Phys. Plasmas 16, 043110 (2009).

${ }^{27}$ E. G. Souza, A. Endler, F. B. Rizzato, and R. Pakter, Phys. Rev. Lett. 109, 075003 (2012).

${ }^{28}$ T. P. Coffey, Phys. Fluids 14, 1402-1406 (1971).

${ }^{29}$ T. C. Marshall, Free-Electron Lasers (Macmillan Publishing Company, New York, 1985).

${ }^{30}$ C. Brau, Free-Electron Lasers (Academic Press, London, 1990).

${ }^{31}$ J. A. Sellwood, J. Comput. Phys. 50, 337 (1983).

${ }^{32}$ J. A. Byers and M. Grewal, Phys. Fluids 13, 1819-1830 (1970).

${ }^{33}$ E. G. Evstatiev, P. J. Morrison, and W. Horton, Phys. Plasmas 12, 072108 (2005). 